\title{
A CHARACTERIZATION OF COMBINATORIAL DEMAND
}

\author{
CHRISTOPHER P. CHAMBERS AND FEDERICO ECHENIQUE \\ Abstract. We prove that combinatorial demand functions are \\ characterized by two properties: continuity and the law of demand.
}

\section{INTRODUCTION}

We prove that combinatorial demand functions are characterized by two properties: continuity and the law of demand. Suppose given a finite collection of items. We are interested in the demand for packages, or bundles of items. For each vector of item prices, we are given a collection of demanded packages, and we want to know if there exists a valuation function for packages such that the demanded packages are optimal. Utility is quasilinear in money. So the valuation has to be such that, for each price vector, the demanded packages maximize the value of the packages when one subtracts the sum of the prices for the items in the package.

The two properties that characterize optimal combinatorial demand are upper hemicontinuity and the law of demand. The continuity property is technical, but familiar. The law of demand captures the economic nature of our problem. Demand for a single item must "slope down," meaning that higher prices correspond to smaller demands. For combinatorial demand, the law of demand says that the change in demanded items should have a negative value, when evaluated by the change in prices. The law of demand is an aggregate, or average,

Date: March 21, 2018.

Chambers is at the Department of Economics, University of California at San Diego. Echenique is at the Division of the Humanities and Social Sciences, California Institute of Technology. We wish to thank Itai Sher, Jay Sethuraman, and Renato Paes Leme for discussions on an earlier draft. 
version of the downward sloping demand property, and it has a long history in economics (see, for example, Samuelson (1948)).

In addition to our characterization of combinatorial demand functions, we show that utility functions are uniquely identified by combinatorial demand. Specifically, we show that, up to an additive constant, a unique monotone utility function can be backed out from demand behavior.

While very natural, our result appears to be new. A long literature investigates the combinatorial demands that satisfy specific behavioral properties, such as gross substitutes: Murota (2003), Tamura (2004), and Paes Leme (2014) survey the literature. Our result is more basic, in that we seek to understand optimal demand behavior alone, without additional behavioral properties. Brown and Calsamiglia (2007) investigate a similar question to ours in the context of bundles of infinitely divisible goods, but their result does not extend to combinatorial demand. Sher and il Kim (2014) show that aggregate combinatorial demand genericall identifies individual valuation functions. Finally, we should mention the paper by Baldwin and Klemperer (2012) which introduces a new framework for the study of discrete demand, investigates many of its properties, and their implications for markets for discrete goods.

Our main result (Theorem 11) follows along the lines of Rochet's approach to revealed preference theory (see Rochet (1987)). The property of cyclic monotonicity is crucial to obtain a rationalizing valuation. We use the results of Lavi et al. (2003) or Saks and Yu (2005) (in a version due to Ashlagi et al. (2010)) to establish that the law of demand is sufficient for cyclic monotonicity. The main issue in adapting these various results to our problem is that cyclic monotonicity is not enough to obtain a strict rationalization: the difficulty is that one may add optimal packages when constructing the rationalization from cyclic monotonicity. The crucial idea to overcome this difficulty is contained in Lemma 5 in the proof.

Our result on identification (Theorem 3) is essentially an adaptation of Theorem 24.9 in Rockafellar (1970). 


\section{Results}

2.1. Notation: Let $X$ be a finite set. Let $S$ be the set of all nonempty subsets of $2^{X}$ (so the empty set is not in $S$, but $\{\varnothing\}$ is).

We identify a set $A \subseteq X$ with its indicator function $1_{A} \in \mathbf{R}^{X}$. The inner product of a vector $p \in \mathbf{R}^{X}$ and $1_{A}$ is denoted by $\langle p, A\rangle=$ $\sum_{x \in A} p_{x}$.

2.2. Rationalizable demand. A demand function is a function $D$ : $\mathbf{R}_{++}^{X} \rightarrow S$ with the property that there is $\bar{p} \in \mathbf{R}_{++}^{X}$ such that $D(p)=$ $\{\varnothing\}$ for all $p \geq \bar{p}$.

The relevant properties for a demand function are three: A demand function $D$

- is quasilinear rationalizable if there exists $v: 2^{X} \rightarrow \mathbf{R}$ such that

$$
D(p)=\operatorname{argmax}\{v(A)-\langle p, A\rangle: A \subseteq X\} ;
$$

- satisfies the law of demand if for all $p, q \in \mathbf{R}_{++}^{X}$, and all $A \in$ $D(p)$ and $B \in D(q)$,

$$
\langle p-q, A-B\rangle \leq 0
$$

- is upper hemicontinuous if, for all $p \in \mathbf{R}_{++}^{X}$, there is a neighborhood $V$ of $p$ such that $D(q) \subseteq D(p)$ when $q \in V$.

Theorem 1. A demand function is quasilinear rationalizable iff it is upper hemicontinuous and satisfies the law of demand.

A stronger condition places more restrictions on the rationalization. We say a function $g: \mathbf{R}_{+}^{X} \rightarrow \mathbf{R}$ is monotone if for all $x, y \in \mathbf{R}_{+}^{X}, x \leq y$ (coordinatewise) implies $g(x) \leq g(y)$. $D$ is monotone, concave, quasilinear rationalizable ( $M C Q$-rationalizable) if there exists a monotone, concave $g: \mathbf{R}_{+}^{X} \rightarrow \mathbf{R}$ such that $v(A)=g\left(1_{A}\right)$, and

$$
D(p)=\operatorname{argmax}\{v(A)-\langle p, A\rangle: A \subseteq X\} .
$$

An easy corollary, demonstrated by our proof is the following:

Corollary 2. If a demand function is quasilinear rationalizable, then it is $M C Q$-rationalizable. 
The corollary demonstrates that there is no additional empirical content delivered by the hypotheses of concavity and monotonicity.

2.3. Identification. Say that $v: 2^{X} \rightarrow \mathbf{R}$ (quasilinear) rationalizes $D$ if for all $p \in \mathbf{R}_{++}^{X}, D(p)=\arg \max _{A \in S} v(A)-\langle p, A\rangle$.

If $v$ rationalizes $D$, then so does $v+c$ for any constant function $c$. So one can only hope to obtain identification up to an additive constant, and that is indeed what one obtains.

We say that $v$ is monotone if for all $A, B \in S$, if $A \subseteq B$, then $v(A) \leq v(B)$. It is easy to see (see Remark 9 below) that if $D$ is quasilinear rationalizable then there is a monotone $v$ that quasilinear rationalizes it.

Theorem 3. For any quasilinear rationalizable $D$, there is a unique monotone $v$ for which $v(\varnothing)=0$ which rationalizes $D$.

\section{Proof of Theorem 1}

Lemma 4. If $D$ is quasilinear rationalizable then it is upper hemicontinuous and satisfies the law of demand

Proof. Let $v$ rationalize $B$. Let $u(p)=\max \{v(A)-\langle p, A\rangle: A \subseteq X\}$.

First we show that $D$ is upper hemicontinuous. Since $X$ is finite, there is $\varepsilon>0$ such that $u(p)-\left(v\left(B^{\prime}\right)-\left\langle p, B^{\prime}\right\rangle\right)>\varepsilon$ for all $B^{\prime} \notin D(p)$. Let $V$ be a ball with center $p$ and radius small enough that for all $q \in V$, and all $B^{\prime} \notin D(p), u(q)-\left(v\left(B^{\prime}\right)-\left\langle q, B^{\prime}\right\rangle\right)>\varepsilon$. Then $D(q) \subseteq D(p)$ for all $q \in V$.

Second we show the law of demand. Let $A \in D(p)$ and $A^{\prime} \in D\left(p^{\prime}\right)$. Then $v(A)-\langle p, A\rangle \geq v\left(A^{\prime}\right)-\left\langle p, A^{\prime}\right\rangle$ and $v\left(A^{\prime}\right)-\left\langle p^{\prime}, A^{\prime}\right\rangle \geq v(A)-\left\langle p^{\prime}, A\right\rangle$. Adding these two inequalities and rearranging yields $\left\langle p-p^{\prime}, A-A^{\prime}\right\rangle \leq$ 0 .

Lemma 4 establishes the necessity direction in the theorem. We now turn to sufficiency. The upper hemicontinuity of $D$ implies the following property: A demand function $D$ satisfies condition $\boldsymbol{\phi}$ if for all $p$ and $B \notin D(p)$ there is $A \in D(p)$ and $p^{\prime}$ such that $A \in D\left(p^{\prime}\right)$ and $\left\langle p^{\prime}, A-B\right\rangle>\langle p, A-B\rangle$. 
Lemma 5. If $D$ is upper hemicontinuous, then it satisfies condition

Proof. Let $p \in \mathbf{R}_{++}^{X}$ and $B \notin D(p)$. Let $V$ be a neighborhood of $p$ as in the definition of upper hemicontinuity. So $D(q) \subseteq D(p)$ for all $q \in V$.

Let $W=\cup_{A^{\prime} \in D(p)}\left(A^{\prime} \backslash B\right)$ and $E=\cup_{A^{\prime} \in D(p)}\left(B \backslash A^{\prime}\right)$. Note that

$$
\left(B \backslash A^{\prime}\right) \cup\left(A^{\prime} \backslash B\right) \neq \varnothing
$$

for each $A^{\prime} \in D(p)$.

Let $\lambda, \lambda^{\prime}>0$. By definition of $W,\left\langle 1_{W}, B\right\rangle=0$. So

$$
\left\langle\lambda 1_{W}-\lambda^{\prime} 1_{E}, A^{\prime}-B\right\rangle=\lambda\left\langle 1_{W}, A^{\prime}\right\rangle-\lambda^{\prime}\left\langle 1_{E}, A^{\prime}\right\rangle+\lambda^{\prime}\left\langle 1_{E}, B\right\rangle .
$$

Then for each $A^{\prime} \in D(p)$, (1) implies that $\left\langle 1_{W}, A^{\prime}\right\rangle \neq 0$ or $\left\langle 1_{E}, B\right\rangle \neq 0$, or both. Moreover, if $\left\langle 1_{W}, A^{\prime}\right\rangle=0$ then it must be true that $A^{\prime} \subsetneq B$, which implies that

$$
-\left\langle 1_{E}, A^{\prime}\right\rangle+\left\langle 1_{E}, B\right\rangle=\left\langle 1_{E}, B-A^{\prime}\right\rangle>0 .
$$

Choose $\lambda, \lambda^{\prime}>0$ such that $\lambda\left\langle 1_{W}, A^{\prime}\right\rangle-\lambda^{\prime}\left\langle 1_{E}, A^{\prime}\right\rangle+\lambda^{\prime}\left\langle 1_{E}, B\right\rangle>0$ for all $A^{\prime} \in D(p)$. This is possible by equation (2), and for example by letting $\lambda / \lambda^{\prime}>|X|$. Also choose $\lambda, \lambda^{\prime}$ such that $p^{\prime}=p+\left(\lambda 1_{W}-\lambda^{\prime} 1_{E}\right) \in$ $V$.

Now, for any $A^{\prime} \in D\left(p^{\prime}\right)$,

$$
\left\langle p^{\prime}, A^{\prime}-B\right\rangle-\left\langle p, A^{\prime}-B\right\rangle=\left\langle\left(\lambda 1_{W}-\lambda^{\prime} 1_{E}\right), A^{\prime}-B\right\rangle>0 .
$$

Moreover, $A^{\prime} \in D(p)$, as $p^{\prime} \in V$ and thus $D\left(p^{\prime}\right) \subseteq D(p)$.

A demand function satisfies cyclic monotonicity if, for all $n$, and using summation $\bmod n$,

$$
\sum_{i=1}^{n}\left\langle p_{i}, A_{i}-A_{i+1}\right\rangle \leq 0,
$$

where $A_{i} \in D\left(p_{i}\right)$, for all sequences $\left\{p_{i}\right\}_{i=1}^{n}$.

The following argument is mostly standard, adapting the construction of Rockafellar (1966) and Rochet (1987). A potential novelty is the use of the upper hemicontinuity condition in guaranteeing strict inequalities when necessary. 
Lemma 6. If D satisfies cyclic monotonicity, and condition $\mathbf{A}$, then it is quasilinear rationalizable.

Proof. We have assumed that there is $p^{*}$ for which $\{\varnothing\}=D\left(p^{*}\right)$. For any $A \subseteq X$, define:

$$
v(A)=\inf \left\langle p_{1}, A-A_{1}\right\rangle+\ldots+\left\langle p^{*}, A_{k}-\varnothing\right\rangle,
$$

where the infimum is taken over all finite sequences $\left(p_{i}, A_{i}\right)_{i=1}^{k}$ for which $A_{i} \in D\left(p_{i}\right)$.

Observe that by cyclic monotonicity, $v(\varnothing) \in \mathbf{R}$; in fact $v(\varnothing) \geq$ 0 . By construction, $v$ is nondecreasing, as it is the lower envelope of nondecreasing functions. Hence $v(A) \in \mathbf{R}$ for all $A$. Finally, observe that $v$ is the lower envelope of restriction of affine functions on $\mathbf{R}^{X}$. Conclude that $v$ is the restriction of a concave function on $\mathbf{R}^{X}$.

Finally, observe by construction that if $A \in D(p)$, then for any $B \subseteq X$

$$
v(B) \leq\langle p, B-A\rangle+v(A),
$$

from which we obtain $v(A)-\langle p, A\rangle \geq v(B)-\langle p, B\rangle$.

Finally, to prove the lemma we need to show that if in addition $B \notin D(p)$ then $v(A)-\langle p, A\rangle>v(B)-\langle p, B\rangle$, or that $v(A)>\langle p, A-$ $B\rangle+v(B)$. By condition $\boldsymbol{\phi}$, there is $A^{\prime} \in D(p)$ and $p^{\prime}$ such that $A^{\prime} \in D\left(p^{\prime}\right)$ and $\left.\left\langle p^{\prime}, A^{\prime}-B\right\rangle\right\rangle\left\langle p, A^{\prime}-B\right\rangle$.

Suppose that $\left\{\left(A_{i}, p_{i}\right)\right\}$ is a sequence as in the definition of $v\left(A^{\prime}\right)$. Then

$v(B) \leq\left\langle p^{\prime}, B-A^{\prime}\right\rangle+\sum_{i=1}^{n}\left\langle p_{i}, A_{i}-A_{i+1}\right\rangle<\left\langle p, B-A^{\prime}\right\rangle+\sum_{i=1}^{n}\left\langle p_{i}, A_{i}-A_{i+1}\right\rangle$, so $v(B)<\left\langle p, B-A^{\prime}\right\rangle+v\left(A^{\prime}\right)$; and thus

$$
v(A)-\langle p, A\rangle=v\left(A^{\prime}\right)-\left\langle p, A^{\prime}\right\rangle>v(B)-\left\langle p^{\prime}, B\right\rangle .
$$


We finish the proof by using a recent result in the mechanism design literature, establishing conditions under which monotonicity (a condition that coincides with the law of demand) implies cyclic monotonicity: see Lavi et al. (2003) and Saks and Yu (2005).

Lemma 7. A demand function satisfies cyclic monotonicity if it satisfies the law of demand.

Proof. So let $D$ satisfy the law of demand and suppose towards a contradiction that there is a sequence $\left(p_{i}, A_{i}\right)_{i=1}^{n}$, with $A_{i} \in D\left(p_{i}\right)$ and $\sum_{i=1}^{n}\left\langle p_{i}, A_{i}-A_{i+1}\right\rangle>0$ (summation $\bmod n$ ), but no such sequence with $n \leq 2$. Choose such a sequence with minimal $n$, and observe that $n \geq 3$.

For any selection $f(p) \in D(p)$, if $f$ is monotone then it is cyclically monotone, see e.g. Saks and Yu (2005) or Ashlagi et al. (2010), Theorem S.7 in the supplementary material 1 Since $D$ satisfies the law of demand, any selection $f$ is monotone, and therefore cyclically monotone.

If $p_{i} \neq p_{j}$ for all $i, j=1, \ldots, n$ with $i \neq j$, then we can choose a selection $f$ of $D$ with $f\left(p_{i}\right)=A_{i}$, violating cyclic monotonicity of $f$, and hence contradicting the fact that it is monotone.

We now claim that in fact it is the case that $p_{i} \neq p_{j}$ for all $i \neq j$.

Observe first that if $p_{i}=p_{i+1}$ for some $i$, then $\left\langle p_{i} A_{i}-A_{i+1}\right\rangle+$ $\left\langle p_{i+1} A_{i+1}-A_{i+2}\right\rangle=\left\langle p_{i} A_{i}-A_{i+2}\right\rangle$, implying the existence of a shorter sequence, a contradiction.

Suppose then that $p_{i}=p_{j}$. By the preceding, we know that $j=i+1$ is false, and $i=j+1$ is false. Without loss, suppose that $i=1$. Then $j \neq n$ and $j \neq 2$. Further, $\left\langle p_{j}, A_{j}-A_{j+1}\right\rangle=\left\langle p_{j}, A_{j}-A_{1}\right\rangle+\left\langle p_{1}, A_{1}-\right.$

\footnotetext{
${ }^{1}$ Technically, the Ashlagi et al. (2010) result requires the output of $f$ to be a probability measure. To modify the construction to fit our environment, simply let $y^{*} \notin X$, and consider the set $Y \subseteq \mathbf{R}^{X \cup\left\{y^{*}\right\}}$ given by $Y=\left\{(p, 0): p \in \mathbf{R}_{++}^{X}\right\}$. Define the function $f^{*}: Y \rightarrow \Delta\left(X \cup\left\{y^{*}\right\}\right)$ by $f^{*}(p, 0)(x)=\frac{1_{x \in f(p)}}{|X|}$ and $f^{*}(p, 0)\left(y^{*}\right)=$ $1-\frac{|f(p)|}{|X|}$. Observe that $\left\langle(q, 0), f^{*}(p, 0)\right\rangle=\langle q, f(p)\rangle \frac{1}{|x|}$, and therefore monotonicity of $f$ is equivalent to that of $f^{*}$ and cyclic monotonicity of $f$ is equivalent to that of $f^{*}$.
} 


$$
\begin{aligned}
& \left.A_{j+1}\right\rangle \text {, so } \\
& 0<\sum_{i=1}^{n}\left\langle p_{i}, A_{i}-A_{i+1}\right\rangle=\left\langle p_{1}, A_{1}-A_{2}\right\rangle+\cdots+\left\langle p_{j}, A_{j}-A_{1}\right\rangle+\left\langle p_{1}, A_{1}-A_{j+1}\right\rangle \\
& +\left\langle p_{j+1}, A_{j+1}-A_{j+2}\right\rangle+\cdots+\left\langle p_{n}, A_{1}-A_{n}\right\rangle .
\end{aligned}
$$

Consequently, either $\left\langle p_{1}, A_{1}-A_{2}\right\rangle+\cdots+\left\langle p_{j}, A_{j}-A_{1}\right\rangle>0$ or $\left\langle p_{1}, A_{1}-\right.$ $\left.A_{j+1}\right\rangle+\cdots+\left\langle p_{j+1}, A_{j+1}-A_{j+2}\right\rangle+\cdots+\left\langle p_{n}, A_{1}-A_{n}\right\rangle>0$. In either case, we have demonstrated the existence of a shorter cycle violating cyclic monotonicity, a contradiction.

\section{Proof of Theorem 3}

As usual, $D\left(\mathbf{R}_{++}^{X}\right)=\bigcup_{p \in \mathbf{R}_{++}^{X}} D(p)$ is the range of $D$, and similarly for a function $f: \mathbf{R}_{++}^{X} \rightarrow 2^{X}$.

First, we characterize those bundles which are demanded at some set of prices.

Lemma 8. Given $v$ which rationalizes $D, A \in D\left(\mathbf{R}_{++}^{X}\right)$ iff for all $B \subset A, B \neq A, v(B)<v(A)$.

Proof. First suppose that $A \in D(p)$ for some $p$. Then for all $B \subset A$, $B \neq A, v(A)-\langle p, A\rangle \geq v(B)-\langle p, B\rangle$, implying $v(A) \geq v(B)+\langle p, A-$ $B\rangle>v(B)$.

Conversely, suppose that for all $B \subseteq A, B \neq A$, we have $v(B)<$ $v(A)$. We want to show that there is $p^{*}$ for which $v(A)-\left\langle p^{*}, A\right\rangle \geq$ $v(B)-\left\langle p^{*}, B\right\rangle$. To ensure this inequality is satisfied, we simply choose $p^{*}(x)$ small for $x \in A$ and large for $x \notin A$.

Remark 9. By Lemma 8, it is straightforward to see that for any $h$ : $S \rightarrow \mathbf{R}$, there is a monotone $\bar{h}$ which rationalizes the same demand as $h$, namely, $\bar{h}$ is the smallest monotone function pointwise dominating $h$ :

$$
\bar{h}(A)=\sup _{B \subseteq A} h(B) .
$$


4.1. Proof of Theorem 3. We proceed to show that if $v$ and $w$ are monotone and both rationalize $D$, they differ by a constant.

For all $p \in \mathbf{R}_{++}^{X}$, let $f(p) \in D(p)$. First we show that any two functions that rationalize $D$ must differ by a constant on the range of $f$. So let $v$ rationalize $D$. Define $U_{v}(p)=\sup _{A \in S} v(A)-\langle p, A\rangle$ (the indirect utility function). Observe that by definition, $U_{v}(p) \geq v(A)-\langle p, A\rangle$ for all $(p, A)$, and $A \in D(p)$ iff $v(A)-\langle p, A\rangle=U(p)$. Consequently, $v(A) \leq U_{v}(p)+\langle p, A\rangle$ for all $(p, A)$, with equality iff $A \in D(p)$. It follows that $A \in D(p)$ iff for all $q, U_{v}(p)+\langle p, A\rangle \leq U_{v}(q)+\langle q, A\rangle$; i.e. $U_{v}(q) \geq U_{v}(p)+\langle p-q, A\rangle$; or $U_{v}(q) \geq U_{v}(p)+\langle q-p,-A\rangle$. In other words, $-A$ is a subgradient of $U_{v}$ at $p$ iff $A \in D(p)$. In particular, $f(p)$ is a subgradient of $U_{v}$.

Observe that $U_{v}$ is convex, real-valued, and continuous, and defined on $\mathbf{R}_{++}^{X}$, an open domain. Since $U_{v}$ is convex, for any $x_{1}, x_{2}$, the function $h(\lambda)=U_{v}\left(\lambda x_{2}+(1-\lambda) x_{1}\right)$ is convex. Since $f$ is a subgradient of $U_{v}$ at each $p$, we obtain that

$$
\begin{aligned}
h(\lambda+\delta)-h(\lambda) & =U_{v}\left(\lambda x_{2}+(1-\lambda) x_{1}+\delta\left(x_{2}-x_{1}\right)\right)-U_{v}\left(\lambda x_{2}+(1-\lambda) x_{1}\right) \\
& \geq\left\langle f\left(\lambda x_{2}+(1-\lambda) x_{1}\right), \delta\left(x_{2}-x_{1}\right)\right\rangle \\
& =\delta\left\langle f\left(\lambda x_{2}+(1-\lambda) x_{1}\right),\left(x_{2}-x_{1}\right)\right\rangle .
\end{aligned}
$$

Hence, $(h(\lambda+\delta)-h(\lambda)) / \delta \geq\left\langle f\left(\lambda x_{2}+(1-\lambda) x_{1}\right),\left(x_{2}-x_{1}\right)\right\rangle$ if $\delta>0$ and $(h(\lambda+\delta)-h(\lambda)) / \delta \leq\left\langle f\left(\lambda x_{2}+(1-\lambda) x_{1}\right),\left(x_{2}-x_{1}\right)\right\rangle$ if $\delta<0$. Thus, $h_{-}^{\prime}(p) \leq f(p) \cdot\left(x_{2}-x_{1}\right) \leq h_{+}^{\prime}(p)$

Observe also that $\int_{0}^{1} h_{-}^{\prime}(x) d x=\int_{0}^{1} h_{+}^{\prime}(x) d x$, by Rockafellar (1970) Corollary 24.2.1. So in particular $U_{v}\left(x_{2}\right)=U_{v}\left(x_{1}\right)+\int_{0}^{1} f(p) \cdot\left(x_{2}-x_{1}\right)$. Recall that $f$ is an arbitrary selection from $D$ and does not depend on $v$. Suppose that $w$ rationalizes $D$, and define $U_{w}$ analogously to $U_{v}$. Then we obtain that

$$
U_{v}\left(x_{2}\right)-U_{v}\left(x_{1}\right)=\int_{0}^{1} f(p) \cdot\left(x_{2}-x_{1}\right)=U_{w}\left(x_{2}\right)-U_{w}\left(x_{1}\right) .
$$

Thus $U_{v}=U_{w}+c$, for some constant $c$.

Now for all $A \in f\left(\mathbf{R}_{++}^{X}\right), v(A)=\inf _{p} U_{v}(p)+\langle p, A\rangle=\inf _{p} U_{w}(p)+$ $\langle p, A\rangle+c=w(A)+c$. So we have shown that $v$ and $w$ differ at most 
by a constant on the range of $f$. Again, since $f$ was arbitrary, $v$ and $w$ differ at most by a constant on the range of $D$.

Recalling that there is always $p^{*} \in \mathbf{R}_{++}^{X}$ for which $\varnothing \in D\left(p^{*}\right)$, by Lemma 8 and monotonicity of $v$, for any $A$ not in the range of $D$, we have

$$
v(A)=\sup _{\left\{B \subset A: B \in D\left(\mathbf{R}_{++}^{X}\right)\right\}} v(B) .
$$

A similar equality holds for $w$, hence $v$ and $w$ differ by a constant.

Therefore there is a unique monotone $v$ with $v(\varnothing)=0$ which rationalizes $D$.

\section{REFERENCES}

Ashlagi, I., M. Braverman, A. Hassidim, and D. Monderer (2010): "Monotonicity and implementability," Econometrica, 17491772.

Baldwin, E. AND P. Klemperer (2012): "Tropical geometry to analyse demand," Tech. rep., Working paper, Oxford University.

Brown, D. J. And C. CAlsamiglia (2007): "The nonparametric approach to applied welfare analysis," Economic Theory, 31, 183188.

Lavi, R., A. Mu'Alem, And N. Nisan (2003): "Towards a characterization of truthful combinatorial auctions," in Foundations of Computer Science, 2003. Proceedings. 44th Annual IEEE Symposium on, IEEE, 574-583.

Murota, K. (2003): Discrete convex analysis, SIAM.

Paes Leme, R. (2014): "Gross substitutability: an algorithmic survey," Preprint.

Rochet, J.-C. (1987): "A necessary and sufficient condition for rationalizability in a quasi-linear context," Journal of Mathematical Economics, 16, 191-200.

RockAfEllar, R. (1966): "Characterization of the subdifferentials of convex functions," Pacific Journal of Mathematics, 17, 497-510.

Rockafellar, R. T. (1970): Convex analysis, Princeton university press. 
SAKS, M. AND L. YU (2005): "Weak monotonicity suffices for truthfulness on convex domains," in Proceedings of the 6th ACM conference on Electronic commerce, ACM, 286-293.

SAmuelson, P. A. (1948): Foundations of economic analysis, Harvard University Press.

SHER, I. AND K. IL KIM (2014): "Identifying combinatorial valuations from aggregate demand," Journal of Economic Theory, 153, 428-458.

TAmurA, A. (2004): "Applications of discrete convex analysis to mathematical economics," Publications of the Research Institute for Mathematical Sciences, 40, 1015-1037. 